\title{
On a note of convergence theorems for zeros of generalized Lipschitz $\Phi$-quasi-accretive operators
}

\author{
Zhigun Xue and Guiwen Lv
}




\title{
ON A NOTE OF CONVERGENCE THEOREMS FOR ZEROS OF GENERALIZED LIPSCHITZ $\Phi$-QUASI-ACCRETIVE OPERATORS
}

\author{
ZHIQUN XUE AND GUIWEN LV
}

Received 17 May, 2012

\begin{abstract}
In this paper, the convergence of Mann iterative process with errors for generalized Lipschitz $\Phi$-quasi-accretive operators is proved in uniformly smooth Banach spaces. Our results improve the corresponding results of Chidume et al.[2].
\end{abstract}

2010 Mathematics Subject Classification: 47H10; 47H09

Keywords: generalized Lipschitz, $\Phi$-quasi-accretive operator, Mann iterative process with errors, fixed point, uniformly smooth Banach space

\section{INTRODUCTION}

Let $E$ be a real Banach space and $E^{*}$ be its dual space. The normalized duality mapping $J: E \rightarrow 2^{E^{*}}$ is defined by

$$
J(x)=\left\{f \in E^{*}:<x, f>=\|x\|^{2}=\|f\|^{2}\right\},
$$

for all $x \in E$, where $\langle\cdot, \cdot>$ denotes the generalized duality pairing. It is well known that

(1) If $E$ is a smooth Banach space, then the mapping $J$ is single-valued.

(2) $J(\alpha x)=\alpha J(x)$ for all $x \in E$ and $\alpha \in \Re$.

(3) If $E$ is a uniformly smooth Banach space, then the mapping $J$ is uniformly continuous on any bounded subset of $E$ (see [1] and [3]).

In the sequel, we denote the single-valued normalized duality mapping by $j$.

Definition 1 ([2]). Let $T: E \rightarrow E$ be an operator. $T$ is said to be strongly accretive if there is a positive constant $k \in(0,1)$ such that for every $x, y \in E$, there exists $j(x-y) \in J(x-y)$ such that

$$
<T x-T y, j(x-y)>\geq k\|x-y\|^{2} .
$$

Let $\tau$ denote the class of all strictly increasing continuous function $f:[0,+\infty) \rightarrow$ $[0,+\infty)$ with $f(0)=0$. Given $\phi \in \tau$, we say that $T$ is $\phi$-strongly accretive if for each $x, y \in E$, there exists $j(x-y) \in J(x-y)$ such that

$$
<T x-T y, j(x-y)>\geq \phi(\|x-y\|)\|x-y\| .
$$

(c) 2015 Miskolc University Press 
Further, let $\Phi \in \tau, T$ is called $\Phi$-accretive if there exists $j(x-y) \in J(x-y)$, such that the inequality

$$
<T x-T y, j(x-y)>\geq \Phi(\|x-y\|)
$$

holds for all $x, y \in E$.

It is shown that the class of $\Phi$-accretive operators not only properly includes the class of $\phi$-strongly accretive operators, but also that of strongly accretive operators. Let $N(T)=\{x \in E: T x=0\} \neq \varnothing$. If the above inequalities (1.1), (1.2) and (1.3) hold for any $x \in E$ and $y \in N(T)$, then the corresponding operator $T$ is called strongly quasi-accretive, $\phi$-strongly quasi-accretive and $\Phi$-quasi-accretive, respectively. Closely related to the class of accretive operators is that of pseudocontractive types.

Let $F(T)=\{x \in E: T x=x\} \neq \varnothing$. A mapping $T: E \rightarrow E$ is called strongly hemi-contractive, $\phi$-strongly hemi-contractive and $\Phi$-hemi-contractive if and only if $I-T$ is strongly quasi-accretive, $\phi$-strongly quasi-accretive and $\Phi$-quasi-accretive, respectively. Here $I$ denotes the identity mapping of $E$.

Definition 2 (see [7]). For arbitrary given $x_{0} \in E$, Mann iterative process with errors $\left\{x_{n}\right\}_{n=0}^{\infty}$ defined by

$$
x_{n+1}=\left(1-a_{n}-c_{n}\right) x_{n}+a_{n} T x_{n}+c_{n} u_{n}, n \geq 0,
$$

where $\left\{u_{n}\right\}$ is any bounded sequence in $E ;\left\{a_{n}\right\}$ and $\left\{c_{n}\right\}$ are two real sequences in $[0,1]$ satisfying $a_{n}+c_{n} \leq 1$ for any $n \geq 0$.

Definition 3 (see [8,9]). A mapping $T: E \rightarrow E$ is called generalized Lipschitz if there exists a constant $L>0$ such that

$$
\|T x-T y\| \leq L(1+\|x-y\|)
$$

for all $x, y \in E$.

Recently, C. E. Chidume and C. O. Chidume [2] established an approximation theorem for the zeros of generalized Lipschitz $\Phi$-quasi-accretive operators. Their result is as follows.

Chidume's Theorem. Let $E$ be a uniformly smooth real Banach space and $A: E \rightarrow$ $E$ be a mapping with $N(A) \neq \varnothing$. Suppose $A$ is a generalized Lipschitz generalized $\Phi$-quasi-accretive mapping. Let $\left\{a_{n}\right\},\left\{b_{n}\right\}$ and $\left\{c_{n}\right\}$ be real sequences in $[0,1]$ satisfying the following conditions: (i) $a_{n}+b_{n}+c_{n}=1$; (ii) $\Sigma_{n=0}^{\infty}\left(b_{n}+c_{n}\right)=\infty$; (iii) $\Sigma_{n=0}^{\infty} c_{n}<\infty$; (iv) $\lim _{n \rightarrow \infty} b_{n}=0$. Let $\left\{x_{n}\right\}$ be generated iteratively from arbitrary $x_{0} \in E$ by,

$$
x_{n+1}=a_{n} x_{n}+b_{n} S x_{n}+c_{n} u_{n}, n \geq 0,
$$

where $S: E \rightarrow E$ is defined by $S x:=f+x-A x, \forall x \in E$ and $\left\{u_{n}\right\}$ is an arbitrary bounded sequence in $E$. Then, there exists $\gamma_{0} \in \Re$ such that if $b_{n}+c_{n} \leq \gamma_{0}, \forall n \geq 0$, the sequence $\left\{x_{n}\right\}$ converges strongly to the unique solution of the equation $A u=0$. 
This result improves a lot of recent contributions in the area. However, there exists a gap in its provided proof. Precisely, $c_{n}=\min \left\{\frac{\epsilon}{4 \beta}, \frac{1}{2 \sigma} \Phi\left(\frac{\epsilon}{2}\right) \alpha_{n}\right\}$ does not holds in line 14 of Claim 2 of page 248, i.e., $c_{n} \leq \frac{1}{2 \sigma} \Phi\left(\frac{\epsilon}{2}\right) \alpha_{n}$ is a wrong case, and it was applied to the formula of line 3rd of page 249 .

Example 1. Setting the iteration parameters: $a_{n}=1-b_{n}-c_{n}$, where

$\left\{b_{n}\right\}_{n=0}^{\infty}: b_{0}=b_{1}=0, b_{n}=\frac{1}{n}, n \geq 2$.

$\left\{c_{n}\right\}_{n=0}^{\infty}: 0, \frac{1}{\sqrt{1^{2}}}, \frac{1}{2^{2}}, \frac{1}{3^{2}}, \frac{1}{\sqrt{4^{2}}}, \frac{1}{5^{2}}, \cdots, \frac{1}{8^{2}}, \frac{1}{\sqrt{9^{2}}}, \frac{1}{10^{2}}, \cdots, \frac{1}{15^{2}}, \frac{1}{\sqrt{16^{2}}}, \frac{1}{17^{2}}, \cdots$.

Then $\Sigma_{n=0}^{\infty} c_{n}<+\infty$, but $c_{n} \neq o\left(b_{n}+c_{n}\right)$. Therefore, the proof of Theorem 3.1 of [2] is incorrect.

The aim of this paper is to establish a convergence result relative to the Mann iteration with errors for generalized Lipschitz $\Phi$-quasi-accretive operators in uniformly smooth real Banach spaces. The following auxiliary facts will be needed.

Lemma 1 (see [3]). Let $E$ be a uniformly smooth real Banach space and let $J$ : $E \rightarrow 2^{E^{*}}$ be a normalized duality mapping. Then

$$
\|x+y\|^{2} \leq\|x\|^{2}+2<y, J(x+y)>
$$

for all $x, y \in E$.

Lemma 2 (see [6]). Let $\left\{\rho_{n}\right\}_{n=0}^{\infty}$ be a nonnegative real numbers sequence satisfying the condition

$$
\rho_{n+1} \leq\left(1-\theta_{n}\right) \rho_{n}+o\left(\theta_{n}\right), n \geq 0,
$$

where $\theta_{n} \in[0,1]$ with $\Sigma_{n=0}^{\infty} \theta_{n}=\infty$. Then $\rho_{n} \rightarrow 0$ as $n \rightarrow \infty$.

\section{RESULTS}

Theorem 1. Let $E$ be an arbitrary uniformly smooth real Banach space and $T$ : $E \rightarrow E$ be a generalized Lipschitz $\Phi$-quasi-accretive operator with $N(T) \neq \varnothing$. Let $\left\{a_{n}\right\},\left\{c_{n}\right\}$ be two real numbers sequences in $[0,1]$ and satisfy the conditions $(i) a_{n}+$ $c_{n} \leq 1$; (ii) $a_{n}, c_{n} \rightarrow 0$ as $n \rightarrow \infty$ and $c_{n}=o\left(a_{n}\right)$; (iii) $\Sigma_{n=0}^{\infty} a_{n}=\infty$. For some $x_{0} \in E$, let $\left\{u_{n}\right\}$ be any bounded sequence and $\left\{x_{n}\right\}$ be Mann iterative sequence with errors defined by

$$
x_{n+1}=\left(1-a_{n}-c_{n}\right) x_{n}+a_{n} S x_{n}+c_{n} u_{n}, n \geq 0,
$$

where $S: E \rightarrow E$ is defined by $S x=x-T x$ for any $x \in E$. Then sequence $\left\{x_{n}\right\}$ converges strongly to the unique solution of the equation $T x=0$.

Proof. Let $q \in N(T)$, that is $q \in F(S)$. Since $T: E \rightarrow E$ is a generalized Lipschitz $\Phi$-quasi-accretive operator, then $S$ is a generalized Lipschitz $\Phi$-hemi-contractive, i.e., there exists $\Phi \in \tau$ such that

$$
<S x-S q, J(x-q)>\leq\|x-q\|^{2}-\Phi(\|x-q\|),
$$


and

$$
\|S x-S y\| \leq L(1+\|x-y\|)
$$

for any $x, y \in E$.

Step 1: There exists $x_{0} \in D$ and $x_{0} \neq S x_{0}$ such that

$$
r_{0}=\left\|x_{0}-S x_{0}\right\| \cdot\left\|x_{0}-q\right\| \in R(\Phi) \text {. }
$$

Indeed, if $\Phi(r) \rightarrow+\infty$ as $r \rightarrow+\infty$, then $r_{0} \in R(\Phi)$; if $\sup \{\Phi(r): r \in[0,+\infty)\}=$ $r_{1}<+\infty$, then for $q \in E$, there exists a sequence $\left\{w_{n}\right\}$ in $E$ such that $w_{n} \rightarrow q$ as $n \rightarrow \infty$ with $w_{n} \neq q$. Furthermore, we obtain that $\left\{w_{n}-S w_{n}\right\}$ is bounded. Hence there exists a natural number $n_{0}$ such that $\left\|w_{n}-S w_{n}\right\| \cdot\left\|w_{n}-q\right\|<\frac{r_{1}}{2}$ for $n \geq n_{0}$, then we renew define $x_{0}=w_{n_{0}}$ and $\left\|x_{0}-S x_{0}\right\| \cdot\left\|x_{0}-q\right\| \in R(\Phi)$.

Step 2: For any $n \geq 0,\left\{x_{n}\right\}$ is bounded.

Setting $R=\Phi^{-1}\left(r_{0}\right)$, then from Definition 2, we obtain that $\left\|x_{0}-q\right\| \leq R$. Denote

$$
B_{1}=\{x \in D:\|x-q\| \leq R\}, B_{2}=\{x \in D:\|x-q\| \leq 2 R\} .
$$

Since $S$ is generalized Lipschitz, so $S$ is bounded. Let

$$
M=\sup _{x \in B_{2}}\{\|S x-q\|+1\}+\sup _{n}\left\{\left\|u_{n}-q\right\|\right\} .
$$

Next, we want to prove that $x_{n} \in B_{1}$. If $n=0$, then $x_{0} \in B_{1}$. Now assume that it holds for some $n$, i.e., $x_{n} \in B_{1}$. We prove that $x_{n+1} \in B_{1}$. Suppose it is not the case, then $\left\|x_{n+1}-q\right\|>R$. Since $J$ is uniformly continuous on bounded subset of $E$, then for $\epsilon_{0}=\frac{\Phi\left(\frac{R}{2}\right)}{4[L+(1+L) R]}$, there exists $\delta>0$ such that $\|J x-J y\|<\epsilon$ when $\|x-y\|<\delta, \forall x, y \in B_{2}$. Now denote

$$
\tau_{0}=\min \left\{\frac{R}{2 M}, \frac{\Phi\left(\frac{R}{2}\right)}{8 R(M+2 R)}, \frac{\delta}{2(M+2 R)}, \frac{R+L(1+R)}{2(M+R)}\right\} .
$$

Since $a_{n}, c_{n} \rightarrow 0$ as $n \rightarrow \infty$, without loss of generality, we assume that $0 \leq a_{n}, c_{n} \leq$ $\tau_{0}$ for any $n \geq 0$. Since $c_{n}=o\left(a_{n}\right)$, denote $c_{n}<a_{n} \tau_{0}$. So we have

$$
\begin{gathered}
\left\|u_{n}-x_{n}\right\| \leq\left\|x_{n}-q\right\|+\left\|u_{n}-q\right\| \leq R+M, \\
\left\|x_{n}-S x_{n}\right\| \leq L+(1+L)\left\|x_{n}-q\right\| \leq L+(1+L) R, \\
\left\|x_{n}-q\right\| \geq\left\|x_{n+1}-q\right\|-a_{n}\left\|S x_{n}-q\right\|-c_{n}\left\|u_{n}-q\right\|>\frac{R}{2}, \\
\left\|x_{n+1}-q\right\| \leq\left(1-a_{n}-c_{n}\right)\left\|x_{n}-q\right\|+a_{n}\left\|S x_{n}-q\right\|+c_{n}\left\|u_{n}-q\right\| \\
\leq R+\tau_{0} M \leq 2 R,
\end{gathered}
$$


and

$$
\begin{aligned}
\left\|\left(x_{n+1}-q\right)-\left(x_{n}-q\right)\right\| & \leq a_{n}\left\|S x_{n}-x_{n}\right\|+c_{n}\left\|u_{n}-x_{n}\right\| \\
& \leq a_{n}\left(\left\|S x_{n}-q\right\|+\left\|x_{n}-q\right\|\right) \\
& +c_{n}\left(\left\|u_{n}-q\right\|+\left\|x_{n}-q\right\|\right) \\
& \leq \tau_{0}(M+2 R) \\
& <\delta .
\end{aligned}
$$

So

$$
\left\|J\left(x_{n+1}-q\right)-J\left(x_{n}-q\right)\right\|<\epsilon_{0} .
$$

Using Lemma 1 and above formulas, we obtain

$$
\begin{aligned}
\left\|x_{n+1}-q\right\|^{2} & \leq\left\|x_{n}-q\right\|^{2}+2 a_{n}<S x_{n}-x_{n}, J\left(x_{n+1}-q\right)> \\
& +2 c_{n}<u_{n}-x_{n}, J\left(x_{n+1}-q\right)> \\
& \leq\left\|x_{n}-q\right\|^{2}-2 a_{n} \Phi\left(\left\|x_{n}-q\right\|\right)+2 a_{n}\left\|x_{n}-S x_{n}\right\| \\
& \cdot\left\|J\left(x_{n+1}-q\right)-J\left(x_{n}-q\right)\right\| \\
& +2 c_{n}\left\|u_{n}-x_{n}\right\| \cdot\left\|x_{n+1}-q\right\| \\
& \leq R^{2}-2 a_{n} \Phi\left(\frac{R}{2}\right)+2 a_{n}(L+(1+L) R) \epsilon_{0} \\
& +4 a_{n} \tau_{0}(M+R) R \\
& \leq R^{2}
\end{aligned}
$$

which is a contradiction. Hence $x_{n+1} \in B_{1}$, i.e., $\left\{x_{n}\right\}$ is a bounded sequence.

Step 3: We want to prove that $\left\|x_{n}-q\right\| \rightarrow 0$ as $n \rightarrow \infty$.

Setting

$$
M_{1}=\sup _{n}\left\|x_{n}-q\right\|+\sup _{n}\left\|u_{n}-q\right\| .
$$

Since $a_{n}, c_{n} \rightarrow 0$ as $n \rightarrow \infty$, then

$$
\left\|\left(x_{n+1}-q\right)-\left(x_{n}-q\right)\right\| \rightarrow 0 .
$$

Hence

$$
\left\|J\left(x_{n+1}-q\right)-J\left(x_{n}-q\right)\right\| \rightarrow 0
$$


as $n \rightarrow \infty$. From (2.7), we have

$$
\begin{aligned}
\left\|x_{n+1}-q\right\|^{2} & \leq\left\|x_{n}-q\right\|^{2}-2 a_{n} \Phi\left(\left\|x_{n}-q\right\|\right) \\
& +2 a_{n}\left\|x_{n}-S x_{n}\right\| \cdot\left\|J\left(x_{n+1}-q\right)-J\left(x_{n}-q\right)\right\| \\
& +2 c_{n}\left\|u_{n}-x_{n}\right\| \cdot\left\|x_{n+1}-q\right\| \\
& \leq\left\|x_{n}-q\right\|^{2}-2 a_{n} \Phi\left(\left\|x_{n}-q\right\|\right) \\
& +2 a_{n}\left[(1+L)\left\|x_{n}-q\right\|+L\right] A_{n} \\
& +2 c_{n}\left\|u_{n}-x_{n}\right\| \cdot\left\|x_{n+1}-q\right\| \\
& \leq\left\|x_{n}-q\right\|^{2}-2 a_{n} \Phi\left(\left\|x_{n}-q\right\|\right) \\
& +a_{n} A_{n}(1+L)\left\|x_{n}-q\right\|^{2}+a_{n} A_{n}(1+L) \\
& +2 a_{n} L A_{n}+4 c_{n} M_{1}^{2} \\
& \leq\left\|x_{n}-q\right\|^{2}-2 a_{n} \Phi\left(\left\|x_{n}-q\right\|\right) \\
& \left.+a_{n} A_{n}(1+L)\right) M_{1}^{2}+a_{n} A_{n}(1+3 L)+4 c_{n} M_{1}^{2} \\
& =\left\|x_{n}-q\right\|^{2}-2 a_{n} \Phi\left(\left\|x_{n}-q\right\|\right)+C_{n} \\
& =\left\|x_{n}-q\right\|^{2}+2 a_{n}\left[B_{n}-\Phi\left(\left\|x_{n}-q\right\|\right)\right],
\end{aligned}
$$

where

$$
\begin{gathered}
A_{n}=\left\|J\left(x_{n+1}-q\right)-J\left(x_{n}-q\right)\right\|, \quad B_{n}=\frac{C_{n}}{2 a_{n}}, \\
\left.C_{n}=a_{n} A_{n}(1+L)\right) M_{1}^{2}+a_{n} A_{n}(1+3 L)+4 c_{n} M_{1}^{2} .
\end{gathered}
$$

Letting $\inf _{n \geq 0} \frac{\Phi\left(\left\|x_{n}-q\right\|\right)}{1+\left\|x_{n+1}-q\right\|^{2}}=\lambda$, then $\lambda=0$. If it is not the case, we assume that $\lambda>0$. Let $0<\gamma<\min \{1, \lambda\}$, then $\frac{\Phi\left(\left\|x_{n}-q\right\|\right)}{1+\left\|x_{n+1}-q\right\|^{2}} \geq \gamma$, i.e.,

$$
\Phi\left(\left\|x_{n}-q\right\|\right) \geq \gamma+\gamma\left\|x_{n+1}-q\right\|^{2} \geq \gamma\left\|x_{n+1}-q\right\|^{2} .
$$

Thus, from (2.8) that

$$
\left\|x_{n+1}-q\right\|^{2} \leq\left\|x_{n}-q\right\|^{2}+2 a_{n}\left(B_{n}-\gamma\left\|x_{n+1}-q\right\|^{2}\right),
$$

which implies that

$$
\begin{aligned}
\left\|x_{n+1}-q\right\|^{2} & \leq \frac{1}{1+2 a_{n} \gamma}\left\|x_{n}-q\right\|^{2}+\frac{2 a_{n} B_{n}}{1+2 a_{n} \gamma} \\
& =\left(1-\frac{2 a_{n} \gamma}{1+2 a_{n} \gamma}\right)\left\|x_{n}-q\right\|^{2}+\frac{2 a_{n} B_{n}}{1+2 a_{n} \gamma} .
\end{aligned}
$$

Let $\rho_{n}=\left\|x_{n}-q\right\|^{2}, \lambda_{n}=\frac{2 a_{n} \gamma}{1+2 a_{n} \gamma}, \sigma_{n}=\frac{2 a_{n} B_{n}}{1+2 a_{n} \gamma}$. Then we get that

$$
\rho_{n+1} \leq\left(1-\lambda_{n}\right) \rho_{n}+\sigma_{n} .
$$

Applying Lemma 2, then $\rho_{n} \rightarrow 0$ as $n \rightarrow \infty$. Thus $\lambda=0$, it is a contradiction. Therefore, there exists an infinite subsequence such that $\frac{\Phi\left(\left\|x_{n_{i}}-q\right\|\right)}{1+\left\|x_{n_{i}+1}-q\right\|^{2}} \rightarrow 0$ as $i \rightarrow$ 
$\infty$. Since $0 \leq \frac{\Phi\left(\left\|x_{n_{i}}-q\right\|\right)}{1+M_{1}^{2}} \leq \frac{\Phi\left(\left\|x_{n_{i}}-q\right\|\right)}{1+\left\|x_{n_{i}+1}-q\right\|^{2}}$, then $\Phi\left(\left\|x_{n_{i}}-q\right\|\right) \rightarrow 0$ as $i \rightarrow \infty$. By the strictly increasing and continuity of $\Phi$, we get $\left\|x_{n_{i}}-q\right\| \rightarrow 0$ as $i \rightarrow \infty$. Next we prove $\left\|x_{n}-q\right\| \rightarrow 0$ as $n \rightarrow \infty$. Let $\forall \varepsilon \in(0,1)$, there exists $n_{i_{0}}$ such that $\left\|x_{n_{i}}-q\right\|<$ $\epsilon, a_{n}, c_{n}<\frac{\epsilon}{8 M_{1}}, B_{n}<\frac{1}{2} \Phi\left(\frac{\epsilon}{2}\right)$, for any $n_{i}, n \geq n_{i_{0}}$. First, we prove $\left\|x_{n_{i}+1}-q\right\|<\epsilon$. Suppose that it is not this case, then $\left\|x_{n_{i}+1}-q\right\| \geq \epsilon$. By Definition 2, we estimate the following formula:

$$
\begin{aligned}
& \left\|x_{n_{i}}-q\right\| \geq\left\|x_{n_{i}+1}-q\right\|-a_{n_{i}}\left\|S x_{n_{i}}-x_{n_{i}}\right\|-c_{n_{i}}\left\|u_{n_{i}}-x_{n_{i}}\right\| \\
& >\epsilon-a_{n_{i}}\left[\left\|S x_{n_{i}}-q\right\|+\left\|x_{n_{i}}-q\right\|\right]-c_{n_{i}}\left[\left\|u_{n_{i}}-q\right\|+\left\|x_{n_{i}}-q\right\|\right] \\
& \geq \epsilon-\left(b_{n_{i}}+c_{n_{i}}\right) 2 M_{1} \\
& >\frac{\epsilon}{2} .
\end{aligned}
$$

Since $\Phi$ is strictly increasing, (2.11) leads to

$$
\Phi\left(\left\|x_{n_{i}}-q\right\|\right) \geq \Phi\left(\frac{\epsilon}{2}\right) .
$$

From (2.8), we have

$$
\begin{aligned}
\left\|x_{n_{i}+1}-q\right\|^{2} & \leq\left\|x_{n_{i}}-q\right\|^{2}+2 a_{n_{i}}\left[B_{n_{i}}-\Phi\left(\left\|x_{n_{i}}-q\right\|\right)\right] \\
& <\epsilon^{2}+2 a_{n_{i}}\left[\frac{1}{2} \Phi\left(\frac{\epsilon}{2}\right)-\Phi\left(\frac{\epsilon}{2}\right)\right] \\
& \leq \epsilon^{2}
\end{aligned}
$$

which is a contradiction. Thus $\left\|x_{n_{i}+1}-q\right\|<\epsilon$. Suppose that $\left\|x_{n_{i}+m}-q\right\|<\epsilon$ holds. Repeating the above course, we can easily show that $\left\|x_{n_{i}+m+1}-q\right\|<\epsilon$ holds. Therefore, we obtain that $\left\|x_{n_{i}+m}-q\right\|<\epsilon$ for any positive integer $m$, which means $\left\|x_{n}-q\right\| \rightarrow 0$ as $n \rightarrow \infty$. This completes the proof.

Remark 1. In above theorem we assume a condition to have $N(T) \neq \varnothing$ for generalized Lipschitz $\Phi$-quasi-accretive operator. However before presenting the main results, we note that this assumption is not necessary in [4] and [5]. The reason is that for Lipschitz or continuous $\Phi$-accretive operators we have $N(T) \neq \varnothing$, but for a generalized Lipschitz mapping it can not be assumed that it must be Lipschitz or continuous, which means that $N(T)$ may be empty. For this, we add a sufficient condition. Therefore our Theorem 1 includes the past results of [4] and [5] which are known as the existence theorems for Lipschitz or continuous $\Phi$-accretive operators and which are special cases of our Theorem 1.

Theorem 2. Let $D$ be a nonempty closed convex subset of uniformly smooth real Banach space $E$, and $T: D \rightarrow D$ a generalized Lipschitz $\Phi$-hemi-contractive mapping with $q \in F(T) \neq \varnothing$. Let $\left\{a_{n}\right\},\left\{c_{n}\right\}$ be real sequences in $[0,1]$ and satisfy the conditions (i) $a_{n}+c_{n} \leq 1$; (ii) $a_{n} \rightarrow 0$ as $n \rightarrow \infty$; (iii) $\Sigma_{n=0}^{\infty} a_{n}=\infty$; (iv) $c_{n}=o\left(a_{n}\right)$. 
Let $\left\{u_{n}\right\}$ be any bounded sequence in $D$. For some $x_{0} \in D$, let $\left\{x_{n}\right\}$ be Mann iterative scheme with errors defined by (1.4). Then $\left\{x_{n}\right\}$ converges strongly to the unique fixed point $q$ of $T$.

Proof. Since $T: D \rightarrow D$ is a generalized Lipschitz $\Phi$-hemi-contractive mapping, then

$$
T x-T q, J(x-q)>\leq\|x-q\|^{2}-\Phi(\|x-q\|)
$$

and

$$
\|T x-T y\| \leq L(1+\|x-y\|)
$$

hold for any $x, y \in E, q \in F(T)$. The rest follows as in Theorem 1 .

Remark 2. In Theorem 1 and Theorem 2, the condition $\Sigma_{n=0}^{\infty} c_{n}<\infty$ of the iteration parameter $\left\{c_{n}\right\}$ is replaced by $c_{n}=o\left(a_{n}\right)$, and these two conditions are not included each other (See above Counterexample). Up to now, it is unknown whether the results of Chidume et al. [2] hold for the condition $\Sigma_{n=0}^{\infty} c_{n}<\infty$. Hence our Theorem 1 and Theorem 2 improve Theorem 3.1 and Theorem 3.2 of [2], respectively.

\section{ACKNOWLEDGEMENT}

The authors are grateful to the anonymous referees for their appreciation, comments and suggestions which helped the improvement of the paper.

\section{REFERENCES}

[1] F. E. Browder, "Nonlinear operators and nonlinear equations of evolution in Banach spaces," Proc. Sympos. Pure. Math., vol. 18, 1976.

[2] C. E. Chidume and C. O. Chidume, "Convergence theorem for zeros of generalized Lipschitz generalized $\phi$-quasi-accretive operators," Proc. Amer. Math. Soc., vol. 134, no. 1, pp. 243-251, 2006.

[3] K. Deimling, Nonlinear Functional Analysis. Berlin: Springer-Verlag, 1985.

[4] J. A. Park and S. Park, "Surjectivity of $\phi$-accretive operators," Proc. Amer. Math. Soc., vol. 90, no. 2, pp. 289-292, 1984.

[5] M. Turinici, "Solvability results for (strongly) self-accretive maps," An. Şt. Univ. ”Al. I. Cuza" Iaşi (Mat.), vol. 35, pp. 117-129, 1989.

[6] X. Weng, "Fixed point iteration for local strictly pseudocontractive mapping," Proc. Amer. Math. Soc., vol. 113, no. 3, pp. 727-731, 1991.

[7] Y. G. Xu, "Ishikawa and mann iterative processes with errors for nonlinear strongly accretive operator equations," J. Math. Anal. Appl., vol. 224, no. 1, pp. 91-101, 1998.

[8] Z. Q. Xue, H. Y. Zhou, and Y. J. Cho, "Iterative solutions of nonlinear equations for $m$-accretive operators in Banach spaces," J. Nonlinear and Convex Analysis, vol. 1, no. 3, pp. 313-320, 2000.

[9] H. Y. Zhou and D. Q. Chen, "Iterative approximations of fixed points for nonlinear mappings of $\varphi$-hemicontractive type in normed linear spaces," Math. Appl., vol. 11, no. 3, pp. 118-121, 1998. 
Authors' addresses

Zhiqun Xue

Department of Mathematics and Physics, Shijiazhuang Tiedao University, Shijiazhuang, 050043, P.R.China

E-mail address: xuezhiqun@126.com

Guiwen Lv

Department of Mathematics and Physics, Shijiazhuang Tiedao University, Shijiazhuang, 050043, P.R.China

E-mail address: lvguiwenyy@126.com 